\title{
SUI COMPLESSI DI RETTE, CHE AMMETTONO UN GRUPPO CONTINUO PROIETTIVO.
}

\author{
Memoria di Ugo Amaldi (Modena).
}

Aduzanza del 27 gennajo 1907.

Sono gid ben noti parecchi tipi di complessi di rette, che ammettono un gruppo continuo di trasformazioni proiettive in sè stessi. Anche prescindendo dai casi ovvii dei complessi lineari e dei complessi (speciali) delle tangenti alle superficie con infinite trasformazioni proiettive o delle rette incidenti alle curve $W$ di KLEIN-LIE, possiamo ricordare $\mathrm{i}$ complessi tetraedrali e $\mathrm{i}$ tipi di complessi incontrati dal sig. W. DE TANnENBERG, nelle sue interessanti ricerche Sur les équations aux dérivées partielles $d u$ premier ordre à deux variables indépendantes, qui admettent un groupe continu de transformations ${ }^{*}$ ).

Io, nel presente lavoro, determino e classifico tutti i complessi di rette che ammettono un gruppo continuo di omografie a tre parametri almeno ${ }^{* *}$ ); il che, notoriamente, equivale a determinare e classificare, su di una quadrica a quattro dimensioni non specializzata dello spazio a cinque dimensioni, le varietd $\infty^{3}$, che ammettono un gruppo continuo proiettivo.

In questa classificazione, com'è naturale, assegno ad un medesimo tipo, non soltanto tutti $i$ trasformati proiettivi di un complesso, ma anche i rispettivi complessi duali. Giungo cosi a trentaquattro tipi di complessi, fra $\mathrm{i}$ quali son duali di sè stessi tutti gli otto tipi, che ammettono un gruppo proiettivo non integrabile (cfr. $n^{\circ}$ ), e sedici dei ventisei tipi a gruppo integrabile (cfr. $n^{0} 24$ ).

I.

\section{Preliminari.}

I. In generale un gruppo proiettivo $\infty^{3}$ ammette un fascio di complessi invarianti, costituiti ciascuno dalle $\infty^{3}$ trasformate di una retta mediante le trasformazioni del gruppo; mentre d'altro canto è agevolmente riconoscibile il caso, in cui sono invarianti

*) Annales de la Faculté des Sciences de Toulouse, t. V (1891).

*) Il caso di un numero minore di parametri (complessi dipendenti da una funzione arbitraria di uno o due argomenti) è implicitamente esaurito da ricerche ben note. Cfr. LIE, Bestimmung aller Flächen, die eine continuirliche Schaar von projectiven Transformationen gestatten [Leipz. Berichte, t. XLVII (I895), pp. 209-260]. 
rispetto al gruppo tutte le congruenze rettilinee di una rete e, quindi, un'infiniti di complessi, dipendente da una funzione arbitraria di due argomenti.

Siamo cosi naturalmente condotti a prender le mosse dalla classificazione dei gruppi continui proiettivi $\infty^{3}$ dello spazio. Determinati i complessi che ammettono un gruppo siffatto, sarà facile riconoscere quelli, cui spetta un gruppo più ampio.

Ora i gruppi proiettivi $\infty^{3}$ non integrabili (e perciò isomorfi al gruppo proiettivo totale della retta) sono conosciuti in base al ben noto teorema dello STUDY ${ }^{*}$ ); mentre d'altra parte $\mathrm{i}$ gruppi integrabili, in quanto lasciano fermo un piano (e una retta in esso e un punto di questa) ${ }^{* *}$ ), sono tutti proiettivamente equivalenti a gruppi lineari; cosicchè alla loro determinazione si giunge agevolmente partendo dalla determinazione compiuta gia dal LrE dei gruppi lineari omogenei ${ }^{* * *}$ ).

2. Per la determinazione dei complessi invarianti di un gruppo proiettivo $\infty^{3}$, noto in coordinate di punto, è necessario anzitutto tener conto delle relazioni di isomorfismo (oloedrico), fra il gruppo proiettivo totale dello spazio e il gruppo da esso subordinato sulla varieta quadratica $\infty^{4}$ delle rette.

Ora, in vista delle integrazioni che si dovranno eseguire, a noi converrd individuar le rette per mezzo di coordinate non omogenee; precisamente ci varremo delle coordinate del PlücKer; solo modificheremo le notazioni tradizionali, assumendo le equazioni ridotte di una retta, in coordinate cartesiane $x, y$, sotto la forma:

$$
x=z_{1}+z_{2} z, \quad y=z_{3}+z_{4} z
$$

in altre parole designeremo con

$$
z_{1}, z_{2}, z_{3}, z_{4}, z_{5}=z_{1} z_{4}-z_{2} z_{3}
$$

rispettivamente le solite coordinate

$$
\rho, r, \sigma, s, n=s p-r \sigma .
$$

Ricordiamo subito che codeste coordinate sono legate alle coordinate omogenee $p_{i j}$ di Grassmann-Cayley e alle coordinate differenziali del Lie dalle seguenti equazioni :

(2)

$$
\left\{\begin{array}{c}
z_{1}=\frac{p_{31}}{p_{34}}=\frac{x d z-z d x}{d z}, \quad z_{2}=\frac{p_{14}}{p_{34}}=\frac{d x}{d z}, \quad z_{3}=\frac{p_{3^{2}}}{p_{34}}=\frac{y d z-z d y}{d z}, \\
z_{4}=\frac{p_{24}}{p_{34}}=\frac{d y}{d z}, \quad z_{5}=\frac{p_{21}}{p_{34}}=\frac{x d y-y d x}{d z} .
\end{array}\right.
$$

Quanto alle suaccennate relazioni di isomorfismo fra il gruppo proiettivo totale e il gruppo subordinato da esso sulla varieta delle rette, basta che, assumendo le $z_{1}, z_{2}$, $z_{3}, z_{4}$ come coordinate sulla quadrica :

$$
z_{5}=z_{1} z_{4}-z_{2} z_{3}
$$

*) LIE-ENGEL, Theorie der Transformationsgruppen, III. Abschn., pag. 785 .

*) Ibidem, I. Abschn., pag. 589.

***) Ibidem, III. Abschn., Kap. 6, pag. Iog. 
applichiamo i notissimi metodi del LIE. Posto al solito:

$$
\begin{aligned}
p=\frac{\partial f}{\partial x}, \quad q=\frac{\partial f}{\partial y}, \quad r & =\frac{\partial f}{\partial z}, \quad U=x p+y q+z r \\
r_{i} & =\frac{\partial f}{\partial z_{i}} \quad(i=\mathrm{I}, 2,3,4),
\end{aligned}
$$

otteniamo cosi il seguente quadro di relazioni :

(3) $\left\{\begin{array}{lcc}p \equiv r_{1}, & q \equiv r_{3}, & r \equiv-z_{2} r_{1}-z_{4} r_{3}, \\ x p \equiv z_{1} r_{1}+z_{2} r_{2}, \quad x q \equiv z_{1} r_{3}+z_{2} r_{4}, & x r \equiv-z_{1} z_{4} r_{3}-z_{2}\left(z_{1} r_{1}+z_{2} r_{2}+z_{4} r_{4}\right) \\ y p \equiv z_{3} r_{1}+z_{4} r_{2}, & y q \equiv z_{3} r_{3}+z_{4} r_{4}, & y r \equiv-z_{2} z_{3} r_{1}-z_{4}\left(z_{2} r_{2}+z_{3} r_{3}+z_{4} r_{4}\right) \\ z p \equiv r_{2}, & z q \equiv r_{4}, & z r \equiv-z_{2} r_{2}-z_{4} r_{4} \\ x U \equiv z_{2} z_{3} r_{4}+z_{1}\left(z_{1} r_{1}+z_{2} r_{2}+z_{3} r_{3}\right), y q \equiv z_{1} z_{4} r_{2}+z_{3}\left(z_{1} r_{1}+z_{3} r_{3}+z_{4} r_{4}\right), z U \equiv z_{1} r_{2}+z_{3} r_{4} .\end{array}\right.$

3. Conosciuto un gruppo proiettivo $\infty^{3}$ in coordinate di punto, noi anzitutto, per mezzo delle (3), ne esprimeremo le trasformazioni infinitesime in coordinate $z_{i}$ di retta :

$$
X_{i}(f)=\sum_{\mathrm{r}}^{4} \xi_{i j}\left(z_{\mathrm{I}}, z_{2}, z_{3}, z_{4}\right) r_{j} \quad(i=1,2,3) .
$$

Dopo di che, se la matrice

$$
\left\|\xi_{i j}\right\| \quad(i=\mathrm{I}, 2,3 ; j=\mathrm{I}, 2,3,4)
$$

non è identicamente nulla, si otterrà l'equazione di un complesso invariante, uguagliando a zero l'eventuale massimo comun divisore dei determinanti del terz'ordine estratti dalla matrice stessa. Ad ogni modo avremo un fascio di complessi invarianti uguagliando ad una costante arbitraria l'unica soluzione del sistema completo ternario in quattro variabili:

$$
X_{1}(f)=\text { o, } \quad X_{2}(f)=\text { o. } \quad X_{3}(f)=\text { o. }
$$

Se poi la matrice (4) è identicamente nulla, il sistema completo precedente si riduce a binario ${ }^{*}$ ); ed uguagliando a due costanti arbitrarie una coppia di soluzioni indipendenti di esso, si ottengono $\infty^{2}$ congruenze rettilinee invarianti rispetto al gruppo.

II.

\section{Complessi che ammettono un gruppo proiettivo non integrabile.}

4. In base al già citato teorema dello Study i gruppi proiettivi $\infty^{3}$ non integrabili (e quindi oloedricamente isomorfi al gruppo proiettivo totale della retta) si riducono ai seguenti tipi :

a) gruppo che ammette un fascio di piani uniti;

b) gruppo che ammette due rette unite sghembe e quindi lascia ferme tutte le generatrici di un sistema di una quadrica;

c) gruppo che trasforma in sè una conica e un punto fuori del piano di essa (gruppo delle rotazioni intorno ad un punto);

d) gruppo della cubica sgemba.

*) Qui evidentemente non può presentarsi il casó che il sistema si riduca ad una sola equazione. 
5. Il gruppo che lascia fermi tutti i piani passanti per l'asse $x=y=0$ e i punti impropri del piano $z=0$ è dato da

$$
r, z r, \quad z U \equiv z_{2} r_{1}+z_{4} r_{3}, \quad z_{2} r_{2}+z_{4} r_{4}, \quad z_{1} r_{2}+z_{3} r_{4},
$$

onde risulta che il fascio di complessi invarianti è costituito in questo caso dai complessi lineari $\left.{ }^{*}\right)$ :

dove $b$ è una costante arbitraria.

$$
z_{s}=b
$$

Come tipo dei complessi lineari non speciali possiamo assumere il complesso:

$$
z_{s}+1=0 \text {, }
$$

il cui gruppo proiettivo (notoriamente $\infty^{10}$ ) è dato da

$\left[G_{1}\right]$

$$
\left\{\begin{array}{c}
p-y r, q+x r, \quad r, \quad x q, \quad x p-y q, \quad y p, \quad z r+U \\
z p-y U, \quad z q+x U, z U ;
\end{array}\right.
$$

mentre a tipo dei complessi lineari speciali sceglieremo il complesso:

[II]

il quale ammette il gruppo $\infty^{11}$ :

$$
z_{s}=0 \text {, }
$$

$\left[G_{\text {II }}\right]$

$$
r, x p, x q, y p, \quad y q, z p, z q, z r, x U, y U, z U .
$$

6. Il gruppo che lascia fermi una conica e un punto fuori del piano di quella, trasforma in sè le quadriche di un fascio e quindi gli $\infty^{I}$ complessi delle tangenti a codeste quadriche, ciascuno dei quali ammette senz'altro il gruppo proiettivo $\infty^{6}$ della rispettiva quadrica.

Di più il nostro gruppo $\infty^{3}$ trasforma in sè il complesso delle rette incidenti alla conica (doppia) base del fascio delle quadriche invarianti, il quale manifestamente ammette l'intero gruppo $\infty^{7}$ di codesta conica.

Scelto come gruppo $\infty^{3}$ quello delle rotazioni intorno all'origine, potremo assumere a rappresentante dei complessi del primo tipo il complesso delle tangenti alla sfera di raggio I e di centro nell'origine

[III]

$$
I-z_{1}^{2}+z_{2}^{2}-z_{3}^{2}+z_{4}^{2}-z_{5}^{2}=0,
$$

il cui gruppo è

$\left[G_{\mathrm{III}}\right] \quad y r-z q, \quad z p-x r, \quad x q-y p, p-x U, q-y U, \quad r-z U$;

mentre l'altro tipo di complessi invarianti sard qui rappresentato dal complesso delle rette di lunghezza nulla:

[IV]

$$
1+z_{2}^{2}+z_{4}^{2}=0
$$

il quale ammette notoriamente il gruppo dei movimenti euclidei e delle similitudini :

$\left[G_{\mathrm{iv}}\right] \quad p, q, r, y r-z q, \quad z p-x r, x q-y p, x p+y q+z r$.

*) Quando nulla di esplicito si dirà in contrario, si sottintenderà che la matrice (4) non é identicamente nulla e che i suoi determinanti del terz'ordine sono primi fra loro. 
7. Quanto al gruppo che trasforma in sé tutte le generatrici di un sistema di una quadrica, una retta generica dello spazio, appoggiandosi a due di codeste generatrici unite, non può variare, per opera del gruppo, se non nella congruenza lineare che ammette quelle due rette come direttrici; cosicchè sarà invariante rispetto al gruppo ogni complesso, che sia ottenuto associando $\infty^{1}$ di codeste congruenze.

Se prendiamo come quadrica invariante il paraboloide:

$$
z+x y=0
$$

e come generatrici unite le $y=$ cost., il gruppo in parola è dato da

$\left[G_{\mathrm{v}}\right]\left\{\begin{array}{c}p-y r, x p+z r, z q+x U \\ \equiv\left(z_{2} z_{3}+\mathrm{I}\right) r_{1}+z_{4}\left(z_{2} r_{2}+z_{3} r_{3}+z_{4} r_{4}\right), z_{\mathrm{T}} r_{1}-z_{4} r_{4},\left(z_{2} z_{3}+\mathrm{I}\right) r_{4}+z_{1}\left(z_{7} r_{1}+z_{2} r_{2}+z_{3} r_{3}\right) ;\end{array}\right.$

onde risulta che le $\infty^{2}$ congruenze lineari invarianti sono date da

$$
z_{2}=\mu\left(z_{s}+1\right), \quad z_{j}=v\left(z_{s}+1\right) ;
$$

e il gruppo [V] trasformerà in sè ogni complesso:

$[\mathrm{V}]$

$$
\varphi\left(\frac{z_{2}}{z_{s}+1}, \frac{z_{3}}{z_{s}+1}\right)=0
$$

dove $\varphi$ rappresenta una funzione arbitraria dei suoi due argomenti.

$\mathrm{Ci}$ riman da vedere per quali particolari determinazioni della $p$ il complesso [V] ammetta un gruppo a più di tre parametri.

Un gruppo siffatto dovrà sempre trasformare in sè il nostro paraboloide. Ora, mentre il gruppo totale del paraboloide lascia fermo soltanto il complesso delle tangenti, cioè un complesso del tipo [III]; i sottogruppi di esso, che contengono il sottogruppo [V], si riducono manifestamente a tre tipi, secondochè subordinano sulla schiera delle generatrici $y=$ cost. un gruppo $\infty^{2} \circ$ un gruppo $\infty^{1}$ con due generatrici unite distinte oppure coincidenti.

Il primo tipo non dà luogo a nessun complesso nuovo. Negli altri due casi, invece, notiamo che ciascuna delle $\infty^{\mathrm{I}}$ omografie subordinate dal gruppo sulla schiera delle generatrici $y=$ cost. è permutabile con tutte le altre; cosicchè il gruppo trasforma in sè ogni complesso quadratico costituito dalle rette che si appoggiano alle coppie di generatrici corrispondentisi in una determinata fra codeste omografie. A rappresentare codesti due tipi di gruppi possiamo scegliere:

$$
\begin{aligned}
& {\left[G_{\mathrm{v1}}\right] \quad\left\{\equiv \left(\begin{array}{c}
p-y r, x p+z r, z q+x U, y q+z r \\
\equiv\left(z_{2} z_{3}+\mathrm{I}\right) r_{1}+z_{4}\left(\left(z_{2} r_{2}+z_{3} r_{3}+z_{4} r_{4}\right), z_{1} r_{1}-z_{4} r_{4},\right.
\end{array}\right.\right.} \\
& \left(z_{2} z_{3}+1\right) r_{4}+z_{1}\left(z_{1} r_{1}+z_{2} r_{2}+z_{3} r_{3}\right), \quad z_{2} r_{2}-z_{3} r_{3} \text {, } \\
& {\left[G_{\mathrm{vix}}\right]\left\{\begin{array}{c}
p-y r, x p+z r, z q+x U, q-x r \\
\equiv\left(z_{2} z_{3}+\mathrm{I}\right) r_{1}+z_{4}\left(z_{2} r_{2}+z_{3} r_{3}+z_{4} r_{4}\right), z_{2} r_{1}-z_{4} r_{4},\left(z_{2} z_{3}+1\right) r_{4}+z_{1}\left(z_{1} r_{1}+z_{2} r_{2}+z_{3} r_{3}\right), \\
\left(z_{1} z_{4}+1\right) r_{3}+z_{2}\left(z_{1} r_{1}+z_{2} r_{2}+z_{4} r_{4}\right),
\end{array}\right.}
\end{aligned}
$$


cui corrispondono rispettivamente $\mathrm{i}$ fasci di complessi invarianti *) :

[VII]

$$
\begin{gathered}
\left(z_{5}+1\right)^{2}-b z_{2} z_{3}=0, \\
\left(z_{5}+1\right)^{2}++\tau_{2} z_{3}-b z_{2}^{2}=0 .
\end{gathered}
$$

8. Abbiamo infine il gruppo proiettivo $\infty^{3}$ di una-cubica sghemba.

E manifesto a priori che nel fascio dei complessi invarianti un complesso generico sarà costituito dalle $\infty^{3}$ rette, che intersecano la sviluppabile circoscritta alla cubica in quaderne di punti proiettive.

Se la $C^{3}$ è data da

il gruppo è

$$
y=x^{2}, \quad z=x^{3},
$$

$$
\left[G_{\mathrm{vIII}}\right]\left\{\begin{array}{c}
p+2 x q+3 y r, \quad x p+2 y^{\prime} q+3 z r, \quad 3 x U-2 y p-z q \\
\equiv 2\left(z_{1} r_{3}+z_{2} r_{4}\right)+\left(\mathrm{I}-3 z_{2} z_{3}\right) r_{1}-3 z_{4}\left(z_{2} r_{2}+z_{3} r_{3}+z_{4} r_{4}\right), \quad z_{1} r_{1}-2 z_{2} r_{2}+2 z_{3} r_{3}-z_{4} r_{4}, \\
2\left(z_{3} r_{1}+z_{4} r_{2}\right)+\left(\mathrm{I}-3 z_{2} z_{3}\right) r_{4}-3 z_{1}\left(z_{1} r_{1}+z_{2} r_{2}+z_{3} r_{3}\right) .
\end{array}\right.
$$

Per trovare l'equazione generale dei complessi invarianti senza ricorrere alla integrazione del sistema completo, che in questo caso si presenta alquanto malagevole, consideriamo due complessi invarianti particolari: il complesso lineare relativo al sistema nullo definito dalla $C^{3}$, che nel nostro caso è dato da

$$
3 z_{s}-\mathrm{I}=\mathrm{o}
$$

e il complesso cubico delle rette incidenti alla $C^{3}$ :

$$
\left.z_{5}^{3}-2 z_{2} z_{3} z_{5}-z_{1} z_{4} z_{5}+z_{1}^{2} z_{2}+z_{3} z_{4}^{2}-z_{2} z_{3}=0^{* *}\right) \text {. }
$$

Ora se indichiamo rispettivamente con $X_{1}, X_{2}, X_{3}$ le tre trasformazioni infinitesime del $\left[G_{\mathrm{vuI}}\right]$ e con $u, v$ i primi membri delle $(5),(6)$, troviamo:

$$
\begin{aligned}
& X_{1}(u)=3 z_{4} u, \quad X_{2}(u)=0, \quad X_{3}(u)=-3 z_{2} u, \\
& X_{1}(v)=9 z_{4} v, \quad X_{2}(v)=0, \quad X_{3}(v)=-9 z_{2} v,
\end{aligned}
$$

onde risulta, per ogni valore della costante $h$,

$X_{1}\left(v+b u^{3}\right)=9 z_{4}\left(v+b u^{3}\right), X_{2}\left(v+b u^{3}\right)=0, X_{3}\left(v+b u^{3}\right)=-9 z_{2}\left(v+b u^{3}\right)$.

Si conclude quindi che $\mathrm{i}$ complessi invarianti rispetto al gruppo $\left[G_{\mathrm{vIII}}\right]$ sono dati dai complessi cubici :

[VIII] $\left.z_{5}^{3}-2 z_{2} z_{3} z_{5}-z_{1} z_{4} z_{5}+z_{1}^{2} z_{2}+z_{3} z_{4}^{2}-z_{2} z_{3}+b\left(3 z_{5}-1\right)^{j}=0^{* *}\right)$.

9. Riassumendo, $i$ complessi che ammettono un gruppo proiettivo non integrabile appartengono ai seguenti tipi:

*) Codesti complessi coincidono coi complessi $H$ (di $I^{\mathrm{a}}$ e $2^{\mathrm{a}}$ specie) del sig. De TAnnenberg (1. c.), col quale noteremo che essi, nella classificazione di WeIler-Segre, appartengono alle specie [III (III)], [III (I $)$ ].

*) L'espressione al primo membro non è altro che il risultante delle due equazioni cubiche

$$
z_{2} x^{j}-x+z_{3}=0, \quad z_{4} x^{3}-x^{2}+z_{3}=0 \text {. }
$$

***) E pressochè superfluo avvertire che $\mathrm{i}$ resultati di questo numero sono impliciti in ricerche notissime. Per es. cfr. SAlmon (ChemIN): Leçons d'Algèbre supérieure; passim, e in particolare XVII' leçon. 
I) complessi lineari (con gruppo $\infty^{\mathrm{ro}} \mathrm{o} \infty^{\mathrm{II}}$ secondochè il complesso è generale o speciale);

2) complesso quadratico delle rette incidenti ad una conica o complesso delle rette di lungherza nulla (con gruppo $\infty^{i}$ );

3) complesso quadratico delle tangenti a una quadrica (con gruppo $\infty^{6}$ );

4) complessi costituiti da $\infty^{1}$ congruenze lineari le cui direttrici appartengano ad una medesima rigata quairica; il gruppo è in generale $\infty^{3}$ e si amplia, diventando $\infty^{4}$, solo quando le coppie di direttrici si corrispondano in una omografia;

5) complessi cubici delle rette intersecanti in quaderne proiettive di punti una sviluppabile del $4^{\circ}$ ordine (con gruppo $\infty^{3}$ ). Fra questi complessi è compreso come caso limite il complesso delle rette incidenti alla cubica sghemba, spigolo di regresso della sviluppabile.

Non sard inutile avvertire che ciascuno degli otto tipi di complessi sin qui incontrati è duale di sé stesso ${ }^{*}$ ).

III.

\section{Sulla classificazione dei gruppi proiettivi integrabili $\infty^{* * *}$ ).}

ro. Poichè ogni gruppo integrabile lascia fermo un piano, noi, assumendo come piano unito il pian improprio, possiamo anzitutto ricondurci a considerare gruppi lineari (gruppi di affinită). Ma come gid si disse i gruppi lineari omogenei sono stati classificati dal LIE; cosicché a noi qui restano a classificare soltanto quelli non omogenei.

Un gruppo lineare non omogeneo $\infty^{3} G_{3}$ è generato da tre trasformazioni infinitesime della forma:

$$
X_{i}=a_{i} p+b_{i} q+c_{i} r+\bar{X}_{i} \quad(i=1,2,3),
$$

dove le $a_{i}, b_{i}, c$, sono costanti non tutte nulle, e le $\bar{X}_{i}$ sono trasformazioni lineari omogenee, cioè combinazioni lineari omogenee a coefficienti costanti delle

$$
x p, \quad y p, \quad z p, x q, y q, \quad z q, x r, \quad y r, \quad z r .
$$

Le trasformazioni lineari omogenee $\bar{X}_{i}$, ottenute accorciando le $X_{i}$, cioè trascurandone la parte traslatoria 0 di ordine zero, generano da sole un gruppo lineare omogeneo, il quale in sostanza determina il modo, in cui il nostro $G_{\text {; }}$ primitivo opera sul piano improprio. Questo gruppo lineare omogeneo, generato dalle $\bar{X}_{i}$ puó essere $o \infty^{3}$, $o \infty^{2}, o \infty^{1}, o$, addirittura, ridursi alla sola operazione identica; corrispondentemente il nostro gruppo $G$; $\circ$ non conterrà nessuna traslazione infinitesina, oppure ammetterd un sottogruppo di traslazioni $\infty^{1}$ o $\infty^{2}$ o $\infty^{3}$; nel quale ultimo caso il gruppo non potrd essere diverso dal gruppo $p, q, r$ di tutte le traslazioni dello spazio.

*) Notoriamente diconsi duali (o dualistici) due gruppi equivalenti rispetto alla trasformazione di contatto omogenea definita dall'unica equazione $x x^{\prime}+y y^{\prime}+z z^{\prime}+\mathrm{I}=0$.

$\left.{ }^{* *}\right)$ Ctr. LIz-Engel, op. cit., Bè. III, Kap, 13. 
Se allora teniamo conto del fatto che il gruppo $\infty^{3}$ delle traslazioni è invariante nel gruppo lineare totale, vediamo che per classificare i gruppi lineari non omogenei $\infty^{3}$ noi potremo assumere al posto del gruppo accorciato omogeneo $\bar{X}_{i}$ successivamente tutti i gruppi lineari omogenei tipici a tre, a due, a un parametro della tabella di classificazione del LIE; aggiungere nei due ultimi casi rispettivamente una o due traslazioni infinitesime indipendenti; e caso per caso determinare quali termini traslatori o di ordine zero si possano aggiungere alle $\bar{X}_{i}$ considerate, compatibilmente con la proprietd gruppale.

Per questa via, che non richiede se non risoluzioni di sistemi di equazioni lineari, si giunge senza difficoltà alla voluta classificazione, la quale comprende un considerevole numero di tipi. Ma non è necessario che io riproduca qui l'intera tabella; giacchè alcune facili considerazioni dirette ci permettono di eliminar subito, in ordine alla determinazione dei complessi invarianti, buon numero di casi; mentre, d'altro canto, dei gruppi residui solo una parte ci condurrà a tipi di complessi che già non siano stati da noi precedentemente incontrati.

\section{IV.}

\section{Complessi, che ammettono un gruppo proiettivo integrabile contenente più di una traslazione infinitesima.}

I I. Prendendo a considerare i gruppi lineari integrabili $\infty^{3}$ a partire da quelli, che contengono il massimo numero di traslazioni, notiamo che il gruppo $p, q, r$ trasforma in sè ogni stella impropria di rette, e, quindi, ogni complesso (speciale) costituito dalle rette incidenti ad una qualsiasi curva del piano improprio; e, naturalmente, ogni complesso siffatto ammette addirittura il gruppo $\infty^{4}$ delle omotetie, cioè il gruppo

$\left[G_{\mathrm{IX}}\right] \quad p, q, \quad r, U \equiv r_{1}, r_{3}, z_{2} r_{1}+z_{4} r_{3}, z_{1} r_{1}+z_{3} r_{3}$;

mentre, d'altro canto, un tale complesso, la cui equazione sard della forma :

[IX]

$$
\varphi\left(z_{2}, z_{4}\right)=0 \text {, }
$$

dove $\varphi$ rappresenta una funzione qualsiasi dei suoi due argomenti, non ammetterà altre trasformazioni infinitesime all'infuori delle $\left[G_{\mathrm{rx}}\right]$.

Perchè un complesso [IX] ammetta un gruppo più ampio di $\left[G_{\mathrm{Ix}}\right]$ è necessario e sufficiente che la rispettiva curva direttrice sul piano improprio sia una curva $W$ di Kuein-Lie. Ora, esclusi il caso della retta e quello della conica, il quale ultimo ci riconduce al tipo [IV] del complesso delle rette di lunghezza nulla, restano due tipi di curve $W^{*}$ ). Otteniamo cosi due tipi di gruppi $\infty^{5}$ integrabili :

$\left[G_{\mathbf{x}}\right]\left\{\begin{aligned} p, q, r, U, x p+c y q \equiv r_{1}, r_{3}, & z_{2} r_{1}+z_{4} r_{3}, z_{\mathrm{r}} r_{1}+z_{3} r_{3}, z_{1} r_{1}+z_{2} r_{2}+c\left(z_{3} r_{3}+z_{4} r_{4}\right) \\ & \left.\left(c \neq 0, \pm 1,2_{1}\right)_{2}\right),\end{aligned}\right.$
$\left[G_{\mathrm{xI}}\right] p, q, r, U, z p+y q \equiv r_{1}, r_{3}, z_{2} r_{1}+z_{4} r_{3}, z_{1} r_{1}+z_{3} r_{3}, r_{2}+z_{3} r_{3}+z_{4} r_{4}$,

*) Cfr. per es. LIE-EngeL, op. cit., III Absch., Kap. 5. 
ai quali corrispondono rispettivamente $\mathrm{i}$ complessi invarianti

$[\mathrm{X}]$

$[\mathrm{XI}]$

$$
\begin{aligned}
& z_{4}=b z_{2}^{c_{2}}, \\
& z_{4}=b e^{z_{2}} .
\end{aligned}
$$

12. Passando a considerate $i$ gruppi che contengono un sottogruppo $\infty^{2}$ di traslazioni, è facile convincersi che i rispettivi complessi invarianti ammettono l'intero gruppo $\infty^{+}$delle omotetie e perció rientrano nei tipi or ora considerati. Supponiamo, invero, che un complesso ammetta le due traslazioni infinitesime $p$ e $q$, vale a dire tutte le traslazioni parallele al piano $z=0$. Allora tutti $i$ coni del complesso aventi il vertice su di un qualsiasi piano parallelo a codesto piano (le cui generatrici esauriscono l'intero complesso) debbono essere tutti fra loro congruenti; onde risulta senz'altro che essi intersecano il piano improprio secondo una medesima curva e il complesso appartiene al tipo [IX]. Ciò del resto risulta senz'altro dal fatto che, essendo $p \equiv r_{1}, q \equiv r_{3}$, l'equazione del nostro complesso sarà indipendente da $z_{1}, z_{3}$.

Notiamo che alla medesima conclusione si giunge anche partendo dall'ipotesi che il complesso ammetta un qualsiasi gruppo $\infty^{2}$ di omotetie (non costituito da sole traslazioni), come per es. $r, U \equiv z_{2} r_{1}+z_{4} r_{3}, z_{1} r_{1}+z_{3} r_{3}$.

\section{V.}

\section{Complessi che ammettono un gruppo proiettivo integrabile contenente una sola traslazione infinitesima.}

13. Come si disse gid al $\mathrm{n}^{0}$ ıo, per ottenere $\mathrm{i}$ gruppi, di cui qui vogliamo tener parola, dobbiamo considerare successivamente tutti i gruppi lineari omogenei $\infty^{2}$ della tabella del LrE, e aggiungervi una traslazione infinitesima libera, prolungando simultaneamente in tutti $\mathrm{i}$ modi possibili le due trasformazioni omogenee mediante termini di ordine zero.

Ma possiamo subito avvertire che sard inutile prendere in considerazione i gruppi lineari omogenei $\infty^{2}$ aventi un sottogruppo $\infty^{\mathrm{r}}$ di omotetie (cioè la trasformazione infinitesima $U$ ), in quanto ogni gruppo siffatto darà luogo a un gruppo lineare omogeneo $\infty^{3}$ contenente un sottogruppo $\infty^{2}$ di omotetie, e quindi ( $n^{0}$ precedente) a complessi invarianti del tipo [IX].

In secondo luogo gli altri tipi di gruppi lineari non omogenei $\infty^{3}$, ai quali con l'indicato procedimento si giunge, sono, com'è naturale, in parte duale di sè stessi e in parte duali a coppie. Ora noi per ciascuna di codeste coppie di tipi ci limiteremo a considerare un unico rappresentante.

Saranno infine facilmente riconoscibili i gruppi che ddnno luogo a tipi di complessi invarianti, già prima d'ora incontrati (complessi lineari, complessi speciali $[\mathrm{IX}], \ldots$ ).

Applicati questi vari criterî di eliminazione, restano a considerare i seguenti tipi di 
gruppi :
I) $r, x r+a q+b p, y r+c q+b p$
I2) $r, p+x r, y q+c(z r+U)$
2) $r, p-y r, y p+x r+a q$
I3) $r, p+x r, q+x p+2 z r$
3) $r, p-y r, y p+a q$
I4) $r, p+x r, p+y q$
4) $r, q+x r, c x p+y q+(c+1) z r$
5) $r, q+x r, z r-x q+U$
I5) $r, x q+y r, z r-x p+x U$
6) $r, q+x r, x p+z r+a q$
16) $r, x q+y r, z r-x p+q$
7) $r, q+x r, y q+(z+y) r+a p$
I7) $r, x q+y r+p, x p+2 y q+3 z^{r}$
8) $r, x q, a z r+y q+x U$
9) $r, x q, a x p+b z r+q$
Io) $r, x q, z r+a U$
I8) $r, z r+a U, x q+U$
19) $r, z r+a U, y q+b U$
I I) $r, p+x r, z^{r}+U$
20) $r, q+z r, x p+a q$
2I) $r, q+z r, x q+a p$.

14. Il gruppo I) ammette in generale un fascio di complessi invarianti lineari; ma quando sia $a b-b c=0$ il rispettivo sistema completo nelle $z_{i}$ si riduce a binario; scelto a rappresentante di questo tipo particolare il gruppo:

$\left[G_{\mathrm{x} 1 \mathrm{I}}\right]\left\{\begin{array}{c}r, p-y r, q+x r \\ \equiv z_{2} r_{1}+z_{+}{ }^{r},\left(\mathrm{I}+z_{2} z_{3}\right) r_{\mathrm{r}}+z_{4}\left(z_{2} r_{2}+z_{3} r_{3}+z_{4} r_{4}\right),\left(\mathrm{I}-z_{1} z_{4}\right) r_{3}-z_{2}\left(z_{1} r_{1}+z_{2} r_{2}+z_{4} r_{4}\right),\end{array}\right.$ troviamo che esso trasforma in sè agni complesso della rete:

$$
z_{5}+z_{2}+v z_{4}+I=0
$$

questi complessi sono tutti congruenti fra loro (e al complesso $z_{5}+{ }_{1}=0$ ) mediante le $\infty^{2}$ traslazioni parallele al piano $z=0$.

Sard quindi invariante rispetto al gruppo [XII] ogni congruenza che sia intersezione di due dei complessi (7) e quindi ogni complesso costituito da $\infty^{\mathrm{I}}$ siffatte congruenze:

[XII]

$$
\varphi\left(\frac{z_{2}}{z_{5}+1}, \frac{z_{+}}{z_{5}+1}\right)=0
$$

dove $\varphi$ è, al solito, una funzione arbitraria dei suoi due argomenti.

Per determinare i casi particolari del tipo [XII], in cui il gruppo ha più di tre parametri, premettiamo alcune considerazioni. I complessi lineari ( 7 ) hanno tutti comune il fascio delle rette improprie passanti pel punto improprio dell'asse $₹$. Ora ci sarà utile nel seguito l'aver prima considerato senz'altro l'insieme di tutti $i$ complessi lineari cui appartiene codesto fascio, cioè il sistema lineare $\infty^{3}$ di complessi :

$$
\lambda z_{5}+\mu z_{2}+v z_{4}+\mathrm{r}=0 .
$$

Il più ampio gruppo proiettivo che trasformi in sè questo sistema lineare di complessi è dato naturalmente dal gruppo lineare che lascia fermo il punto improprio dell'asse $z$ cioè dal gruppo a dieci parametri 
A noi importa vedere come operi questo gruppo sulla varietà degli $\infty^{3}$ complessi (8). Estendendo, secondo le note regole del LiE, le trasformazioni infinitesime (9) ai parametri $i, \mu, x$ troviamo:

$$
\begin{aligned}
& p-\lambda \frac{\partial f}{\partial \nu}, q+\lambda \frac{\partial f}{\partial \mu}, r, x p-\lambda \frac{\partial f}{\partial \lambda}-\mu \frac{\partial f}{\partial \mu}, x q-\nu \frac{\partial f}{\partial \mu}, \\
& \left(x r-\frac{\partial f}{\partial \mu}, y p-\mu \frac{\partial f}{\partial \nu}, y q-\lambda \frac{\partial f}{\partial \lambda}-v \frac{\partial f}{\partial \nu}, y r-\frac{\partial f}{\partial v}, z r+\lambda \frac{\partial f}{\partial \lambda}+\mu \frac{\partial f}{\partial \mu}+\nu \frac{\partial f}{\partial v}\right. \text {. }
\end{aligned}
$$

Ciò premesso, risulta subito che il più ampio gruppo proiettivo che trasformi in sè la rete $(7)$, cioè la rete $\lambda=\mathrm{I}$, è dato dal gruppo $\infty^{9}$ :

$$
p, q, \quad r, x p-y q, x p+z r, x q, x r, y p, y r
$$

il quale subordina sui complessi $\mu, \nu$ il gruppo:

$$
\frac{\partial f}{\partial \mu}, \quad \frac{\partial f}{\partial \nu}, \quad \mu \frac{\partial f}{\partial \mu}, \quad \mu \frac{\partial f}{\partial v}, \quad \vee \frac{\partial f}{\partial \mu}, \quad \nu \frac{\partial f}{\partial \nu},
$$

cioe il gruppo lineare totale sulle due variabili $\mu$ e $v$.

L'isomorfismo fra i gruppi (I I) e (I2) è meriedrico, in quanto alla identitd del gruppo (12) corrisponde nel gruppo (II) il sottogruppo invariante $\infty^{3}\left[G_{\mathbf{x A z}}\right]$.

Un complesso [XII] si puó rappresentare come una curva del piano $\mu, \nu$; e risulta da quanto s'è detto or ora che un tal complesso ammetterà un gruppo proiettivo a più di tre parametri, quando la rispettiva curva rappresentatrice sul piano $\mu, \nu$ sia trasformata in sè da un gruppo lineare, almeno $\infty^{1}$. E poichè il più ampio gruppo proiettivo che trasformi in sè la rete (7) è isomorfo al gruppo lineare in $\lambda, \mu$, cosi avremo tanti tipi di complessi [XII] a gruppo $\infty^{4}$ (almeno), quanti sono i tipi, distinti rispetto al gruppo lineare, di curve piane con infinite trasformazioni lineari in sè.

Ora risulta da un noto teorema del LIE ${ }^{*}$ ), che le forme tipiche di una trasformazione lineare entro il gruppo lineare sono otto, di cui quattro ammettono traiettorie rettilinee e perció, nel nostro caso, dànno luogo a complessi invarianti lineari. I quattro tipi di trasformazioni infinitesime lineari a traiettorie non lineari sono in coordinate $\mu, v$ :

(13) $\mu \frac{\partial f}{\partial \mu}+c \nu \frac{\partial f}{\partial \nu}\left(c \neq 0\right.$, I), $\mu \frac{\partial f}{\partial \mu}+(\mu+\nu) \frac{\partial f}{\partial \nu}, \frac{\partial f}{\partial \mu}+\nu \frac{\partial f}{\partial \nu}, \frac{\partial f}{\partial \mu}+\mu \frac{\partial f}{\partial \nu}$.

In generale una traiettoria di una qualsiasi delle prime tre trasformazioni infinitesime non ammette nessuna trasformazione lineare ulteriore. Le traiettorie invece della quarta trasformazione sono parabole e, come tali, ammettono ciascuna una ulteriore trasformazione infinitesima lineare; per es. la parabola:

ammette le due trasformazioni :

$$
\mu^{2}=2 \nu
$$

$$
\frac{\partial f}{\partial \mu}+\mu \frac{\partial f}{\partial v}, \quad p \frac{\partial f}{\partial \mu}+2 v \frac{\partial f}{\partial \nu} .
$$

*) Cfr. per es. LIE-SchefFers, Vorlesungen über continuierliche Gruppen, etc., pag. 60. 
Conduce a traiettorie paraboliche anche la prima trasformazione ( 13 ) per $c=2,1 / 2$; onde possiamo escludere questi due casi in quanto conducono a gruppi $\infty^{2}$ del tipo (I 4 ). Possiamo inoltre notare che anche per $c=$ - I la prima trasformazione (13) ha traiettorie coniche; ma in codesto caso la retta impropria non è tangente, cosicchè le coniche non ammettono nessuna ulteriore trasformazione infinitesima lineare.

Concludendo si hanno quattro tipi di complessi [XII], aventi un gruppo più ampio che $\infty^{3}$ : di questi quattro tipi l'uno ammette un gruppo $\infty^{5}$ e gli altri tre un gruppo $\infty^{4}$.

In base alle (Io) troviamo:

$$
\begin{gathered}
\frac{\partial f}{\partial \mu}+\mu \cdot \frac{\partial f}{\partial \nu}, \quad \mu \frac{\partial f}{\partial \mu}+2 v \frac{\partial f}{\partial v} \equiv q+y p, \quad 2 x p+y q+3 z r \\
\mu \frac{\partial f}{\partial \mu}+c \nu \frac{\partial f}{\partial \nu}, \quad\left(c \pm \mathrm{o}, \mathrm{I}, 2,{ }^{\prime} / 2\right) \equiv c x p+y q+(c+\mathrm{I}) z r \\
\mu \frac{\partial f}{\partial \mu}+(\mu+\nu) \frac{\partial f}{\partial \nu} \equiv z r-x q+U \\
\frac{\partial f}{\partial \mu}+\nu \frac{\partial f}{\partial \nu} \equiv x p+z r-q
\end{gathered}
$$

e allora, mediante integrazioni che per brevità tralasciamo, si ottengono i seguenti quattro tipi di complessi ${ }^{*}$ ):

$[\mathrm{XIII}]$
$\left[G_{\mathrm{xUI}}\right]$$\left\{\begin{array}{c}2 z_{4}\left(z_{5}+\mathrm{I}\right)+z_{2}^{2}=0 \\ \equiv z_{2} r_{1}+z_{+} r_{;},\left(\mathrm{I}+z_{2} z_{3}\right) r_{1}+z_{1}\left(z_{2} r_{2}+z_{3} r_{3}+z_{4} r_{4}\right),\left(\mathrm{I}-z_{1} z_{4}\right) r_{3}-z_{2}\left(z_{1} r_{1}+z_{2} r_{2}+z_{4} r_{4}\right) . \\ r_{3}+z_{3} r_{1}+z_{4} r_{2}, 2 z_{1} r_{1}-z_{2} r_{2}+z_{3} r_{3}-2 z_{4} r_{4}\end{array}\right.$

$[\mathrm{XIV}]\left\{\begin{array}{r}z_{2}^{c}=h z_{4}\left(z_{5}+1\right) \\ (c \neq 0,1,2,1 / 2)\end{array}\right.$

$\left.\left[G_{\mathrm{xIV}}\right]\right\}=\begin{gathered}r, p-y r, q+x r, c x p+y q+(c+\mathrm{I}) z r \\ \equiv z_{2} r_{1}+z_{4}{ }^{r}{ }_{3},\left(\mathrm{I}+z_{2} z_{3}\right) r_{1}+z_{4}\left(z_{2} r_{2}+z_{3} r_{3}+z_{4} r_{4}\right),\left(\mathrm{I}-z_{1} z_{4}\right) r_{3}-z_{2}\left(z_{1} r_{1}+z_{2} r_{2}+z_{4}{ }_{4}\right), \\ c z_{1} r_{1}-z_{2} r_{2}+z_{3} r_{3}-c z_{4} r_{4}\end{gathered}$

$[\mathrm{XV}] \int z_{5}+\mathrm{I}=b z_{2} e^{-\frac{z_{4}}{\tilde{z}_{2}}}$

$\left[G_{\mathbf{x v}}\right]\left\{\begin{array}{c}z_{2} r_{1}+z_{4} r_{3},\left(\mathrm{I}+z_{2} z_{3}\right) r_{1}+z_{4}\left(z_{2} r_{2}+z_{3} r_{3}+z_{4} r_{4}\right),\left(1-z_{1} z_{4}\right) r_{3}-z_{2}\left(z_{1} r_{1}+z_{2} r_{2}+z_{4} r_{4}\right), \\ z_{1} r_{1}-z_{2} r_{2}+\left(z_{3}-z_{1}\right) r_{3}-\left(z_{2}+z_{4}\right) r_{4}\end{array}\right.$

$[\mathrm{XVI}] \int z_{4}=b z_{2} e^{\frac{z_{5}+1}{z_{2}}}$

$\left[G_{x v 1}\right]\left\{\begin{array}{c}r, p-y r, q+x r, x p+z r-q \\ \equiv z_{2} r_{1}+z_{4} r_{3},\left(I+z_{2} z_{3}\right) r_{1}+z_{4}\left(z_{2} r_{2}+z_{3} r+z_{4} r_{4}\right),\left(x-z_{1} z_{4}\right) r_{3}-z_{2}\left(z_{1} r_{1}+z_{2} r_{2}+z_{4} r_{4}\right), \\ r-z_{1} r_{1}+z_{4} r .\end{array}\right.$

*) I tipi XIV e XV coincidono coi complessi $K$ (di $1^{a}$ e $2^{a}$ specie) del sig. DE TANnEnberg, 1. c., pag. I03. 
Il complesso quadratico XIII ha come superficie singolare il piano improprio e il punto improprio dell'asse $z$ contati quattro volte; e le rette singolari costituiscono una unica congruenza lineare speciale, avente per asse la retta impropria del piano $y=0$; cosicchè la caratteristica del complesso è $\left.[(32 \mathrm{r})]^{*}\right)$.

Notiamo poi che i gruppi 2), 3), 4), 5),6), 7) sono tutti sottogruppi del gruppo (I I) della rete (8), contenenti ciascuno un sottogruppo $\infty^{2}$ del gruppo $G_{x I I}$; cosicchè essi non possono condurre se non a complessi invarianti appartenenti ai quattro tipi determinati dianzi.

I5. Dopo quanto precede, consideriamo il gruppo 8), cioè $r, x q, a z r+y q+\alpha U \equiv z_{2} r_{1}+z_{4} r_{i}, z_{1} r_{3}+z_{2} r_{4}, 0 z_{1} r_{1}-a z_{2} r_{2}+(I+x) z_{3} r_{3}+(1-a) z_{4} r_{4}$ e, lasciando per un momento da parte i complessi invarianti nel caso generale, notiamo che il rispettivo sistema completo nelle $z_{i}$ si riduce per $a=0, \alpha=-$ I a binario; abbiamo cioè che il gruppo:

$\left[G_{\mathrm{xVII}}\right] \quad r, x q, x p+z r \equiv z_{2} r_{1}+z_{4} r_{3}, \quad z_{1} r_{3}+z_{2} r_{4}, \quad z_{1} r_{1}-z_{4} r_{4}$ trasforma in sè ogni complesso della rete :

$$
\lambda z_{5}+\mu z_{2}+\mathrm{I}=0
$$

(costituita dagli $\infty^{\mathrm{I}}$ complessi $\lambda z_{j}+\mathrm{I}=0$, e dai complessi ad essi congruenti mediante traslazioni parallele all'asse delle $y$ ), e quindi anche ogni complesso:

[XVII]

$$
p\left(z_{2}, z_{s}\right)=0
$$

dove $\varphi$ è, al solito, una funzione arbitraria dei suoi due argomenti.

Per trovare le determinazioni di $p$, per le quali il complesso XVII ammette un gruppo a più di tre parametri, non abbiamo che a valerci dei risultati del $n^{\circ}$ prece- dente. Dalle (Io) risulta anzitutto che il più ampio gruppo proiettivo che trasformi in sè la rete (I5) è il gruppo a sette parametri

$$
q, \quad r, x p, x q, x r, y q, z r
$$

contenente, com'è naturale, il gruppo $G_{\mathrm{xv} \text { s }}$ come sottogruppo invariante.

In secondo luogo dalle medesime formole (IO) discende che il gruppo (I6) subordina sui complessi (IS) il gruppo $\infty^{+}$, ad esso meriedricamente isomorfo,

$$
\lambda \frac{\partial f}{\partial \lambda}, \quad \frac{\partial f}{\partial \mu}, \quad \lambda \frac{\partial f}{\partial \mu}, \quad \mu \frac{\partial f}{\partial \mu},
$$

cioè il gruppo lineare che lascia ferma la retta $\lambda=0$.

Per trovare i complessi XVII aventi un gruppo $\infty^{4}$ almeno, dovremo classificare i tipi di trasformazioni infinitesime del gruppo (I 7 ) entro il gruppo medesimo: al the si giunge con una agevole discussione, che ad ogni modo, rientra in t.na nota discussione del LIE ${ }^{* *}$ ): si ha cosi che una trasformazione infinitesima del gruppo (I 7 ) è equivalente,

*) Cfr. SEGRE, Sulla geometria della retta e delle sue serie quadratiche [Memoria della R. Accademia delle Scienze di Torino, serie II, t. XXXVI, $n^{\circ}$ I68].

*) LIE-SCHEFFERS, Vorlesungen über continuierliche, etc., pag. 60 . 
entro il gruppo stesso, ad una delle cinque seguenti:

$$
\left\{\begin{array}{cl}
\lambda \frac{\partial f}{\partial \lambda}+c \mu \frac{\partial f}{\partial \mu}, \quad \lambda \frac{\partial f}{\partial \lambda}+(\lambda+\mu) \frac{\partial f}{\partial \mu}, & \frac{\partial f}{\partial \mu}+\lambda \frac{\partial f}{\partial \lambda}, \\
\lambda \frac{\partial f}{\partial \lambda}+\mu \frac{\partial f}{\partial \mu}, \quad \mu \frac{\partial f}{\partial \mu}, \quad \lambda \frac{\partial f}{\partial \mu}, & \frac{\partial f}{\partial \mu .} .
\end{array}\right.
$$

Le quattro ultime si possono senz'altro lasciar da parte, in quanto hanno traiettorie rettilinee e, perció, conducono a complessi lineari; e per la stessa ragione si puó supporre nella prima trasformazione infinitesima $c \neq 0$, I. E poi manifesto che qui le traiettorie coniche, alle quali conduce la prima trasformazione infinitesima per $c=2,1 / 2,-\mathrm{I}$ non ammettono, entro il gruppo(17), nessuna altra trasformazione infinitesima. Cosicchè siano condotti a tre tipi di complessi invarianti aventi un gruppo $\infty^{+}$.

Tenendo conto delle (Io) abbiamo:

$\lambda \frac{\partial f}{\partial \lambda}+c \mu \frac{\partial f}{\partial \mu} \equiv(c-1) y q+c z r ; \lambda \frac{\partial f}{\partial \lambda}+(\lambda+\mu) \frac{\partial f}{\partial \mu} \equiv q-x p ; \frac{\partial f}{\partial \mu}+\lambda \frac{\partial f}{\partial \lambda} \equiv y q+x r ;$ onde risulta senz'altro che i gruppi, cui dànno luogo la $\lambda \frac{\partial f}{\partial \lambda}+(\lambda+\mu) \frac{\partial f}{\partial \mu}$ e la $\frac{\partial f}{\partial \mu}+\lambda \frac{\partial f}{\partial \lambda}$ sono fra loro duali. Restano quindi i due seguenti tipi di complessi: $[\mathrm{XVIII}]$
$\left[G_{\mathrm{xVII}}\right]$$\left\{\begin{array}{cc}\tilde{z}_{2}=b z_{5}^{i} & (c \neq 0, \mathrm{r}) \\ r, x q, x p+z r,(c-\mathrm{I}) y q+c z r & \\ \equiv z_{2} r_{1}+z_{4} r_{3}, z_{1} r_{3}+z_{2} r_{4}, z_{1} r_{1}-z_{4} r_{4}, c z_{2} r_{2}-(c-1) z_{3} r_{3}+z_{4} r_{+},\end{array}\right.$

[XIX] $\tilde{z}_{2}=b e^{\frac{z_{5}}{z_{2}}}$ $\left[G_{\mathbf{x i x}}\right]\left\{r, x q, x p+z r, q-x p \equiv z_{2} r_{1}+z_{4} r_{3}, z_{1} r_{3}+z_{2} r_{4}, z_{1} r_{1}-z_{4} r_{4}, r_{3}-z_{1} r_{1}-z_{2} r_{2}\right.$.

Abbiamo con ciò esaurito $i$ tipi di complessi invarianti, cui possono condurre $i$ gruppi 8), 9), Io) della nostra tabella; giacchè codesti gruppi sono tutti sottogruppi del gruppo (I6), aventi, ciaseuno, comune col sottogruppo invariante $G_{\mathbf{x v u}}$ un sottogruppo $\infty^{2}$.

Notiamo infine che il tipo XVIII, in cui il parametro $c$ è essenziale, dà luogo per $c=2,-\mathrm{I}, 1 / 2$ a complessi quadratici :

$$
z_{2}=b z_{5}^{2}, \quad z_{2}=b \sqrt{z_{5}}, \quad z_{2} z_{5}=b
$$

I due primi sono duali fra loro, cosicchè nel tipo XVIII si può supporre $c \neq 1 / 2$, e appartengono alla caratteristica $[(4 \mathrm{II})]$; il terzo appartiene invece alla caratteristica [(2II)(II)]. Nell'uno e nell'altro caso la superficie singolare degenera in due piani (il piano improprio e il piano $x=0$ ) e in due punti ${ }^{*}$ ).

16. Procedendo nello studio dei gruppi della tabella al no ${ }^{0} 3$, notiamo che i gruppi I ), I2), 13), I4) hanno comune il sottogruppo invariante $\infty^{2}$ :

$$
r, p+x r \equiv z_{2} r_{1}+z_{4} r_{3}, r_{1}-z_{1} z_{4} r_{3}-z_{2}\left(z_{1} r_{1}+z_{2} r_{2}+z_{4} r_{4}\right) ;
$$

*) Cfr. Segre, Memoria citata, $\mathbf{n}^{\mathrm{i}}$ 168, 169. 
cosicchè i loro complessi invarianti saranno costituiti ciascuno da $\infty^{1}$ per le $\infty^{2}$ congruenze invarianti del gruppo (19).

Codeste congruenze sono date da

$$
z_{4}=\lambda z_{2}, \quad z_{s} z_{2}-z_{4}=\mu z_{2}^{2}
$$

onde è manifesto che ciascuna di esse si scinde nella stella $z_{2}=z_{4}=0$ delle rette parallele all'asse $z$ e in una congruenza lineare (speciale):

$$
z_{4}=\lambda z_{2}, \quad z_{5}-\mu z_{2}-\lambda=0
$$

Ma a noi gioverà considerare qui, anzichè codeste congruenze, la rete di complessi quadratici :

$$
z_{2} z_{5}-z_{4}+\alpha z_{2} z_{4}+\beta z_{2}^{2}=0
$$

ciascuno dei quali è invariante rispetto al gruppo (Ig). Questi complessi quadratici sono tutti fra loro congruenti mediante le $\infty^{2}$ traslazioni parallele al piano $z=0$ e corrispondono alla caratteristica [(33)]. Per es. il complesso $z_{5} z_{2}-z_{4}=0$ ha come superficie singolare il piano $y^{\prime}=0$ e il punto improprio dell'asse $z$ contati una sol volta $e$ il piano improprio e il punto improprio dell'asse $y$ contati tre volte ${ }^{*}$ ).

Tenendo conto della natura della superficie singolare dei complessi (20), si trova agevolmente che il più ampio gruppo proiettivo che trasformi in sè la rete (2o) è il gruppo $\infty^{6}$ :

$$
p, \quad q, \quad r, \quad x p+2 z r, x r, \quad y q,
$$

le cui trasformazioni infinitesime, estese ai parametri $\alpha, \beta$ della rete diventano:

$$
p-\frac{\partial f}{\partial \alpha}, q+\frac{\partial f}{\partial \beta}, \quad r, x p+2 z r+\alpha \frac{\partial f}{\partial \alpha}, \quad x r+\frac{\partial f}{\partial \alpha}, y q-\beta \frac{\partial f}{\partial \beta} .
$$

Di qui risulta che il gruppo (2I) subordina sulla rete $\alpha, \beta$ il gruppo $\infty^{4}$ ad esso meriedricamente isomorfo :

$$
\frac{\partial f}{\partial \alpha}, \quad \frac{\partial f}{\partial \beta}, \quad \alpha \frac{\partial f}{\partial \alpha}, \beta \frac{\partial f}{\partial \beta},
$$

cioè il gruppo lineare che lascia fermi i punti impropri degli assi $\alpha=0, \beta=0$.

Ogni curva del piano $\alpha$, rappresenta un complesso invariante pel gruppo $\infty^{2}$ (19): per avere i complessi che ammettono un gruppo più ampio, dobbiamo al solito classificare i tipi di trasformazioni infinitesime del gruppo (23) entro il gruppo medesimo. Questi tipi sono, come risulta da una facile discussione :

$$
\begin{gathered}
\frac{\partial f}{\partial \alpha}, \quad \frac{\partial f}{\partial \beta}, \quad \alpha \frac{\partial f}{\partial \alpha}, \quad \beta \frac{\partial f}{\partial \beta} \\
\alpha \frac{\partial f}{\partial \alpha}+c \beta \frac{\partial f}{\partial \beta}(c \neq 0, .1), \frac{\partial f}{\partial \beta}+\alpha \frac{\partial f}{\partial \alpha}, \quad \frac{\partial f}{\partial \alpha}+\beta \frac{\partial f}{\partial \beta} .
\end{gathered}
$$

Se allora teniamo conto delle (22), vediamo che i primi due tipi di trasformazioni infinitesime, in quanto portano nel gruppo una seconda traslazione infinitesima con-

*) Cfr. Segre, ibidem, $\mathrm{n}^{\circ}$ I65.

Rend. Circ. Matem. Palermo, t. XXIII (1907). - Stampato il 2s febbrajo Igo7. 
ducono a complessi speciali del tipo IX: le due successive dànno invece luogo a complessi invarianti appartenenti alla rete (20) e quindi congruenti al complesso quadratico di caratteristica [(33)], di cui già parlammo più sopra:

[XX]

$$
z_{2} z_{5}-z_{4}=0 \text {. }
$$

Il gruppo rispettivo è, come risulta dalle (22), a quattro parametri :

$\left[G_{\mathbf{x x}}\right]\left\{\begin{array}{c}r, p+x r, y q, x p+2 z r \\ \equiv z_{2} r_{1}+z_{4} r_{3}, r_{1}-z_{1} z_{4} r_{3}-z_{2}\left(z_{\mathrm{I}} r_{1}+z_{2} r_{2}+z_{4} r_{4}\right), z_{3} r_{3}+z_{4} r_{4}, z_{1} r_{\mathrm{I}}-z_{2} r_{2}-2 z_{4} r_{4} .\end{array}\right.$ Le ultime tre trasformazioni infinitesime dànno luogo a tre gruppi $\infty^{3}$, ciascuno dei quali caratterizza un tipo di complessi invarianti, che non ammette nessuna ulteriore trasformazione infinitesima:

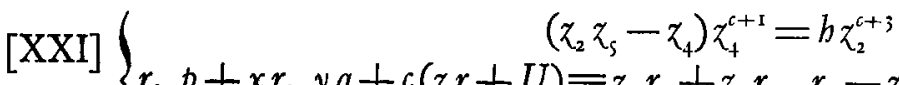

$\left[G_{\mathbf{x x I}}\right]\left\{\begin{array}{c}r, p+x r, y q+c(z r+U) \equiv z_{2} r_{1}+z_{4} r_{3}, r_{1}-z_{1} z_{4} r_{3}-z_{2}\left(z_{1} r_{4}+z_{2} r_{2}+z_{4} r_{4}\right), \\ c\left(z_{1} r_{1}-z_{2} r_{2}\right)+(1+c) z_{3} r_{3}+(\mathrm{I}-c) z_{1} r\end{array}\right.$

$\left[G_{\mathrm{xxII}}\right]\left\{\begin{array}{c}z_{4}=b z_{2} e^{\frac{z_{2} z_{5}-z_{4}}{z_{2}^{2}}} \\ r, p+x r, q+x p+2 z r \equiv z_{2} r_{1}+z_{4} r_{3}, \\ r_{\mathrm{I}}-z_{\mathrm{I}} z_{4} r_{2}-z_{2}\left(z_{\mathrm{I}} r_{\mathrm{I}}+z_{2} r_{2}+z_{4} r_{4}\right), r_{3}+z_{\mathrm{I}} r_{1}-z_{2} r_{2}-2 z_{4} r_{4}\end{array}\right.$

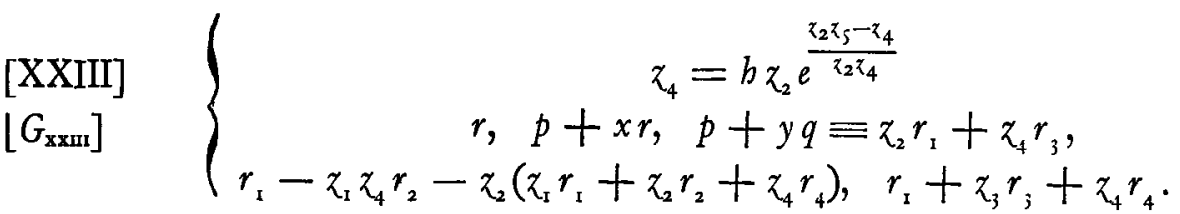

Abbiamo cosi tenuto conto dei gruppi II), I2), I3), I4) della nostra tabella del $\mathrm{n}^{\circ} \mathrm{I} 3$.

17. I gruppi I5) e I6) della solita tabella hanno comune il sottogruppo invariante $\infty^{2}$ :

(24) $\quad r, x q+y r \equiv z_{2} r_{1}+z_{4} r_{3}, \quad z_{1} r_{3}+z_{2} r_{4}-z_{2} z_{3} r_{1}-z_{4}\left(z_{2} r_{2}+z_{3} r_{3}+z_{4} r_{4}\right)$, le cui congruenze invarianti :

$$
z_{s}=\lambda z_{2}, \quad z_{4}^{2}-2 z_{2}=\mu z_{2}^{2}
$$

sono date ciascuna dall'intersezione di un complesso lineare speciale (di asse $x=0$, $\lambda y+\mathrm{I}=0$ ) con un complesso quadratico costituito dalle rette incidenti a una conica del piano improprio.

Il gruppo (24) trasforma quindi in sè ogni complesso quadratico della rete:

$$
\alpha z_{2} z_{5}+2 \beta z_{2}^{2}+z_{4}^{2}-2 z_{2}=0 \text {; }
$$

questa rete di complessi si puó dedurre mediante le $\infty^{\mathrm{r}}$ traslazioni parallele all'asse $y$ dal fascio $\alpha z_{2} z_{5}+z_{4}^{2}-2 z_{2}=0$; e ciascuno di questi complessi, la cui caratteristica è [(33)], appartiene al tipo XIII e percio, ammette un gruppo a cinque parametri.

Il più ampio gruppo proiettivo che trasformi in sè la rete (26) è il gruppo:

$$
q, \quad r, \quad U, \quad x r \quad x q+y r, x p+2 y q+3 z r,
$$


le cui trasformazioni infinitesime, estese ai parametri $\alpha, \beta$ dànno

$$
q+\frac{\mathrm{I}}{2} \alpha \frac{\partial f}{\partial \beta}, r, U-\alpha \frac{\partial f}{\partial \alpha}, x r-\frac{\partial f}{\partial \beta}, x q+y r, x q+2 y q+3 z r+2 \beta \frac{\partial f}{\partial \beta} .
$$

Discende di qui che il gruppo subordinato dal gruppo (27) sui complessi della rete è il gruppo lineare:

$$
\alpha \frac{\partial f}{\partial \alpha}, \quad \frac{\partial f}{\partial \beta}, \quad \alpha \frac{\partial f}{\partial \beta}, \beta \frac{\partial f}{\partial \beta},
$$

che gid incontrammo al $n^{0}$ is.

Dei tipi delle trasformazioni infinitesime del gruppo (28):

$$
\begin{gathered}
c \alpha \frac{\partial f}{\partial \alpha}+\beta \frac{\partial f}{\partial \beta}, \quad \alpha \frac{\partial f}{\partial \alpha}+(\alpha+\beta) \frac{\partial f}{\partial \beta}, \frac{\partial f}{\partial \beta}+\alpha \frac{\partial f}{\partial \alpha}, \\
\alpha \frac{\partial f}{\partial \alpha}+\beta \frac{\partial f}{\partial \beta}, \quad \frac{\partial f}{\partial \beta}, \quad \alpha \frac{\partial f}{\partial \alpha}, \quad \alpha \frac{\partial f}{\partial \beta},
\end{gathered}
$$

gli ultimi quattro dànno luogo a complessi lineari o a complessi speciali del tipo ix. La $\frac{\partial f}{\partial \beta}+\alpha \frac{\partial f}{\partial \alpha}$ conduce al gruppo:

$$
r, x q+y r, x r+U
$$

che, essendo duale di un caso particolare del gruppo 2I) della nostra tabella, che studieremo ben tosto, si puó lasciar da parte. Restano quindi i due primi tipi, che conducono rispettivamente ai gruppi I5) e I6). Nel primo caso si puó supporre $c \neq 0$, giacchè in tale ipotesi $\mathrm{i}$ complessi invarianti sono complessi quadratici della rete (26) e quindi appartenenti al tipo XIII. Si ha, dunque, ponendo $a=2(\mathrm{I}-c)$,

[XXIV]

$\left[G_{\mathbf{x x x}}\right]$

$$
\left\{\begin{array}{c}
z_{2}^{a-1} z_{5}=b\left(z_{4}^{2}-2 z_{2}\right)^{\frac{a}{2}} \\
r, \quad x q+y r, \quad z r-x p+a U \equiv z_{2} r_{1}+z_{4} r_{3}, \\
z_{1} r_{3}+z_{2} r_{4}-z_{2} z_{3} r_{1}-z_{4}\left(z_{2} r_{2}+z_{3} r_{3}+z_{4} r_{4}\right), \\
(a-1) z_{1} r_{1}-2 z_{2} r_{2}+a z_{3} r_{3}-z_{4} r_{4}
\end{array}\right.
$$

$[\mathrm{XXV}]\left\{2 z_{5}=b z_{2} e^{\frac{z_{4}^{2}-2 z_{2}}{z_{2}^{2}}}\right.$

$\left[G_{\mathrm{xxv}}\right]\left\{\begin{array}{c}r, x q+y r, x p-z r+q \equiv z_{2} r_{1}+z_{4} r_{3}, \\ z_{1} r_{3}+z_{2} r_{4}-z_{2} z_{3} r_{1}-z_{4}\left(z_{2} r_{2}+z_{3} r_{3}+z_{4} r_{4}\right), r_{3}+z_{1} r_{1}+2 z_{2} r_{2}+z_{4} r_{4} .\end{array}\right.$

18. Il gruppo 17 ) della nostra tabella del $\mathrm{n}^{\circ} \mathrm{I}_{3}$ :

$$
r, p+x q+y r, x p+2 y q+3 z r
$$

conduce ad un tipo di complessi invarianti gid noto: infatti esso trasforma in sè ogni complesso quadratico del fascio:

$$
z_{2}\left(z_{5}-1\right)=b\left(z_{4}^{2}-2 z_{2}\right)
$$

vale a dire complessi quadratici del tipo XIII.

19. Ci rimangono ancora da considerare i gruppi 18), 19), 20), e 21). 
Tutti questi conducono a complessi che ammettono gruppi a tre soli parametri: possiamo quindi limitarci a dare i risultati.

I gruppi r8), I9) hanno comune il sottogruppo invariante $\infty^{2}$ :

$$
r, \quad z r+a U \equiv z_{2} r_{1}+z_{4} r_{3}, \quad z_{2} r_{2}+z_{+} r_{+}-a\left(z_{1} r_{1}+z_{3} r_{3}\right),
$$

le cui congruenze invarianti :

$$
z_{4}=\lambda z_{2}, \quad z_{2}^{a-1} z_{5}=\mu
$$

sono definite ciascuna come intersezione di un complesso lineare speciale (avente per asse la retta impropria del piano $\lambda x+y^{\prime}=0$ ) con un complesso del tipo XVIII. I rispettivi complessi invarianti, costituiti ciascuno $d a \infty^{1}$ congruenze siffatte, sono dati da

$$
\begin{aligned}
& \text { [XXVI] } \quad\left\{\quad z_{5} z_{2}^{a-s}=b e^{\frac{z_{4}}{z_{2}}}\right.
\end{aligned}
$$

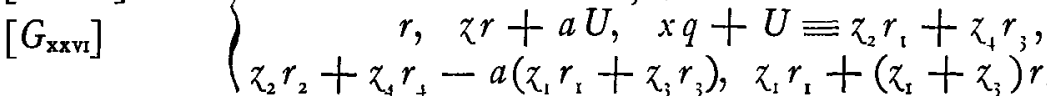

$$
\begin{aligned}
& \left(z_{2} r_{2}+z_{4} r_{4}-a\left(z_{1} r_{1}+z_{3} r_{3}\right), z_{1} r_{1}+\left(z_{1}+z_{3}\right) r_{3}+z_{2} r_{4},\right. \\
& \begin{array}{l}
{[\mathrm{XXVII}]} \\
{\left[G_{\mathrm{xxVII}}\right]}
\end{array}\left\{\begin{array}{c}
z_{5} z_{2}^{a+b}=b z_{4}^{\mathrm{I}+b} \\
r, z r+a U, y q+b U \equiv z_{2} r_{\mathrm{r}}+z_{4} r_{3}, \\
z_{2} r_{2}+z_{4} r_{4}-a\left(z_{1} r_{1}+z_{3} r_{3}\right), z_{3} r_{3}+z_{4} r_{4}+b\left(z_{1} r_{1}+z_{3} r_{3}\right) .
\end{array}\right.
\end{aligned}
$$

Analogamente i gruppi 20) e 21) ammettono entrambi il sottogruppo invariante:

$$
r, q+z r \equiv z_{2} r_{1}+z_{4} r_{3}, r_{3}-z_{2} r_{2}-z_{4} r_{4},
$$

che trasforma in sè ogni congruenza:

$$
z_{4}=\lambda z_{2}, \quad z_{2}=\mu \cdot e^{\frac{z_{5}}{z_{2}}},
$$

intersezione di un complesso lineare speciale con un complesso del tipo XIX. I complessi invarianti dei gruppi 20), 2I) sono, naturalmente, costituiti da $\infty^{3}$ congruenze siffatte :

$$
\begin{aligned}
& \begin{array}{l}
{[\mathrm{XXVIII]}]} \\
{\left[G_{\mathrm{xx} \mathrm{VIII}}\right]}
\end{array}\left\{\begin{array}{c}
z_{4}^{a+1}=b z_{2}^{a} a^{\frac{z_{5}}{\bar{z}_{2}}} \\
r, \quad q+z r, \quad x p+a q \equiv z_{2} r_{1}+z_{4} r_{3}, \quad r_{3}-z_{2} r_{2}-z_{4} r_{4}, \quad z_{1} r_{1}+z_{2} r_{2}+a r_{3},
\end{array}\right. \\
& {[\mathrm{XXIX]})} \\
& {\left[G_{\mathrm{xxx \mathbf {x }}}\right]\left\{\begin{array}{c}
z_{2}^{2}=b e^{z_{2}^{\frac{2}{2}}} \\
r, q+z r, \quad x q+a p \equiv z_{2} r_{1}+z_{4} r_{3}, \quad r_{3}-z_{2} r_{2}-z_{4} r_{4}, \quad z_{1} r_{3}+z_{2} r_{4}+a r_{1} .
\end{array}\right.} \\
& z_{2}^{2}=b e^{\frac{2 z_{2} \tau_{5}-a z_{4}^{2}}{z_{2}^{2}}}
\end{aligned}
$$

VI.

\section{Complessi che ammettono un gruppo proiettivo integrabile $\infty^{3}$ privo di traslazioni infinitesime.}

20. Dobbiamo ancora considerare i gruppi lineari $\infty^{3}$, che ammettono un gruppo accorciato omogeneo a tre parametri. Per determinarli noi non abbiamo che a prolungare in tutti i modi possibili mediante termini traslatori (o di ordine zero) le trasformazioni infinitesime dei varì gruppi lineari omogenei tipici della solita tabella del LIE. Ma esclusi due tipi di gruppi non integrabili che a noi non interessano, è facile rico- 
noscere che buona parte dei gruppi, ai quali cosi si giunge, contengono una trasformazione infinitesima equivalente a una traslazione (cioè, secondo la nomenclatura del LIE, una elazione), e perció appartengono a tipi da noi già prima d'ora incontrati.

Fanno eccezione soltanto i seguenti sottogruppi, fra loro distinti, che soli ci restano oramai da considerare e che ci condurranno a cinque tipi ulteriori di complessi invarianti, fra i quali merita, forse, particolare menzione un complesso del $6^{\circ}$ grado, che è connesso alla rigata cubica del CAYLEY (tipo XXXIV):

a)

b)

c)

d)

e)

1)

g)

b)

$$
\begin{aligned}
& p-y r, \quad x p+z r, \quad y p+a p+b r ; \\
& p-y r, q-x r, \quad(c-1) x p+y q+c z r ; \\
& p-y r, \quad q-x r, \quad r-x p+y q ; \\
& p-y r, \quad x p+z r, \quad y q+z r ; \\
& y r+a p+b q, \quad x r+c p+a q, \quad z r+U ; \\
& x p, \quad y q, \quad z r ; \\
& p+x r, \quad y q, \quad x p+2 z r ; \\
& p+2 x q+3 y r, \quad q+3 x r, \quad x p+2 y q+3 z r .
\end{aligned}
$$

21. Il gruppo a) trasforma in sè soltanto complessi lineari.

I gruppi $b$ ) e $c$ ) hanno comune il sottogruppo invariante $\infty^{2}$ :

$p-y r, q-x r \equiv\left(\mathrm{I}+z_{2} z_{3}\right) r_{\mathrm{x}}+z_{4}\left(z_{2} r_{2}+z_{3} r_{3}+z_{4} r_{4}\right),\left(\mathrm{I}+z_{1} z_{4}\right) r_{3}+z_{2}\left(z_{1} r_{1}+z_{2} r_{2}+z_{4} r_{4}\right)$, il quale trasforma in sè ogni quadrica del fascio

$$
z+x y=\text { cost. }
$$

e ciascuno degli $\infty^{2}$ complessi quadratici del tipo VII, che sono dati ciascuno dalle rette che si appoggiano alle coppie di generatrici di una quadrica (29), corrispondentisi in una omografia parabolica avente come retta unita una delle generatrici improprie del paraboloide.

Questi complessi costituiscono la rete (cfr. il $\mathrm{n}^{0} 7$ ):

$$
4 z_{2} z_{3}+\left(z_{5}+1\right)^{2}+2 \alpha z_{2}^{2}+4 \beta z_{2} z_{4}=0 \text {, }
$$

il cui più ampio gruppo proiettivo è dato da

$$
p-y r, q-x r, \quad r, x p+z r, \quad y q+z r .
$$

Queste trasformazioni infinitesime, estesi ai parametri $\alpha, \beta$ della rete dànno:

$$
p-y r, q-x r, \quad r+\frac{\partial f}{\partial \beta}, \quad x p+z r+\beta \frac{\partial f}{\partial \beta}, \quad y q+z r+\alpha \frac{\partial f}{\partial \alpha}+\beta \frac{\partial f}{\partial \beta} ;
$$

cosicchè il gruppo subordinato dal gruppo (3I) sui complessi della rete è il gruppo $\infty^{3}$ :

$$
\alpha \frac{\partial f}{\partial \alpha}, \frac{\partial f}{\partial \beta}, \beta \frac{\partial f}{\partial \beta},
$$

le cui trasformazioni infinitesime sono reducibili, entro il gruppo stesso, ai seguenti tipi :

$$
\alpha \frac{\partial f}{\partial \alpha}, \quad \frac{\partial f}{\partial \beta}, \quad \beta \frac{\partial f}{\partial \beta}, \quad \alpha \frac{\partial f}{\partial \alpha}+c \beta \frac{\partial f}{\partial \beta}, \quad \frac{\partial f}{\partial \beta}+\alpha \frac{\partial f}{\partial \alpha} .
$$


La $\approx \frac{\partial f}{\partial x}$ conduce al gruppo:

$$
p-y r, q-x r, \quad x p-y q
$$

che trasforma in sè $i$ complessi:

$$
4 z_{2} z_{3}+\left(z_{5}+1\right)^{2}=b z_{2} z_{4},
$$

cioè $i$ complessi delle tangenti alle quadriche (29).

Cosi il gruppo:

$$
p-y r, q-x r, \quad r
$$

cui dà luogo la $\frac{\partial f}{\partial \rho}$ conduce a complessi lineari; e il gruppo:

$$
p-y r, q-x r, x p+z r,
$$

al quale si è condotti dalla $\beta \frac{\partial f}{\partial \beta}$, trasforma in sè complessi quadratici del tipo VII.

Restano dunque le due trasformazioni infinitesime $\alpha \frac{\partial f}{\partial \alpha}+c \beta \frac{\partial \beta}{\partial \beta}$ e $\frac{\partial f}{\partial \beta}+\alpha \frac{\partial f}{\partial \alpha}$, le quali conducono rispettivamente ai nostri gruppi $b$ ) e c) e dànno luogo a due nuovi tipi di complessi, che ammettono non più di $\infty^{3}$ trasformazioni proiettive:

$[\mathrm{XXX}] \int \begin{gathered}z_{2}^{2(c-1)}=b z_{4}^{2}\left[4 z_{2} z_{3}+\left(z_{5}+1\right)^{2}\right]^{c-2} \\ p-y r, q-x r,(c-1) x p+y q+c z r\end{gathered}$

$\left[G_{\mathrm{xxx}}\right] \quad j \equiv\left(\mathrm{I}+z_{2} z_{3}\right) r_{\mathrm{I}}+z_{4}\left(z_{2} r_{2}+z_{3} r_{3}+z_{4} r_{4}\right),\left(\mathrm{I}+z_{\mathrm{I}} z_{4}\right) r_{3}+z_{2}\left(z_{1} r_{1}+z_{2} r_{4}+z_{4} r_{4}\right)$, $(c-I) z_{1} r_{1}-z_{2} r_{2}+z_{3} r_{3}-(c-I) z_{4} r_{+}$.

$[\mathrm{XXXI}]$
$\left[G_{\mathrm{xxxx}}\right]$$\left\{\begin{array}{c}z_{2}^{2}=b z_{4}^{2} e^{\frac{4 z_{2} z_{3}+\left(z_{5}+1\right)^{2}}{z_{2} z_{4}}} \\ p-y r, q-x r, \quad r+y q-x p \\ \equiv\left(\mathrm{I}+z_{2} z_{3}\right) r_{1}+z_{4}\left(z_{2} r_{2}+z_{3} r_{3}+z_{4} r_{4}\right),\left(\mathrm{I}+z_{1} z_{4}\right) r_{3}+z_{2}\left(z_{1} r_{1}+z_{2} r_{2}+z_{4} r_{4}\right), \\ \left(z_{1}+z_{2}\right) r_{1}+z_{2} r_{2}+\left(z_{4}-z_{3}\right) r_{3}-z_{4} r_{4} .\end{array}\right.$

22. I gruppi $d$ ) ed $e$ ) conducono a complessi, appartenenti a tipi già determinati.

Il gruppo $d$ ) trasforma in sè complessi quadratici del tipo VI (cfr. $\mathrm{n}^{\circ} 7$ ); mentre il gruppo $e$ ):

$$
y r+a p+b q, \quad x r+c p+a q, \quad z r+U
$$

se $a^{2}-b c \neq 0$, è equivalente al gruppo:

$$
p-y r, q-x r, z r+U
$$

che ha come invarianti complessi quadratici del tipo VII.

Se poi è $a^{2}-b c=0$ il gruppo si puó ridurre alla forma:

$$
y r, p+x r, z r+U \text {, }
$$

cioè ad un gruppo contenente una elazione; onde risulta che esso non ci puó dare nulla di nuovo.

23. Il gruppo $f$ ):

$\left[G_{x x x I I}\right] \quad x p, \quad y q, \quad z r \equiv z_{1} r_{1}+z_{2} r_{2}, \quad z_{3} r_{3}+z_{4} r_{4}, \quad z_{2} r_{2}+z_{4} r_{4}$ trasforma in sé, oltre il piano improprio, i tre piani coordinati $x=0, y=0, z=0$; 
onde risulta senz'altro che il fascio dei complessi invarianti sarà dato dal fascio dei complessi tetraedrali, relativi a codesti quattro piani invarianti, cioé da

[XXXII]

$$
z_{1} z_{4}=b z_{2} z_{3} \text {. }
$$

I due ultimi gruppi $g$ ) ed $b$ ), che ancora ci restano da considerare, dànno luogo a due nuovi tipi di complessi, rispettivamente del $4^{\circ}$ e del $6^{\circ}$ grado, che, in un certo senso, si possono considerare, al pari del complesso VIII della cubica sghemba, come una generalizzazione del complesso tetraedrale.

Cosi il gruppo $g$ ):

$$
\left[G_{\mathrm{xxxuI}}\right]\left\{\begin{array}{c}
p+x r, y q, x p+2 z r \equiv r_{1}-z_{2} z_{4} r_{3}-z_{2}\left(z_{1} r_{1}+z_{2} r_{2}+z_{4} r_{4}\right) \\
z_{3} r_{3}+z_{4} r_{4}, \quad z_{1} r_{1}-z_{2} r_{2}-2 z_{4} r_{4}
\end{array}\right.
$$

trasforma in sè, oltre il piano improprio, il cilindro quadrico ad esso tangente $2 z-x^{2}=0$ e il piano secante $y=0$. E allora manifesto che il gruppo trasformera in sè ogni complesso, costituito da tutte le rette, che intersecano codesto cilindro e codesti due piani in quaterne di punti, aventi un dato birapporto.

Questi nuovi complessi sono del $4^{\circ}$ grado e ciascuno di essi è individuato da un cono quadrico, da due piani, di cui uno tangente al cono, e dal valore di un parametro (birapporto). Se si ricorda che il gruppo proiettivo di un cono quadrico è $\infty^{\overline{7}}$, si vede tosto che questi complessi non possono anmmettere un gruppo a più di tre parametri.

Il fascio dei complessi invarianti rispetto al gruppo $G_{\mathbf{x x x m}}$ è dato da [XXXIII]

Consideriamo infine il gruppo $b)$ :

$$
\left(\mathrm{I}-2 z_{1} z_{2}\right) z_{4}^{2}=b\left(z_{2} z_{3}-z_{4}\right)^{2}
$$

$$
\left[G_{\mathrm{xxxvv}}\right]\left\{\begin{array}{c}
p+2 x q+3 y r, q+3 x r, x p+2 y q+3 z r \\
\equiv r_{1}+2\left(z_{1} r_{3}+z_{2} r_{4}\right)-3 z_{2} z_{3} r_{1}-3 z_{1}\left(z_{2} r_{2}+z_{3} r_{3}+z_{3} r_{4}\right), \\
\left(\mathrm{I}-3 z_{1} z_{4}\right) r_{3}-3 z_{2}\left(z_{1} r_{1}+z_{2} r_{2}+z_{4} r_{4}\right), z_{1} r_{1}-2 z_{2} r_{2}+2 z_{3} r_{3}-z_{4} r_{4} .
\end{array}\right.
$$

Esso trasforma in sè la rigata cubica del CAYLEY :

$$
z-3 x y+2 x^{3}=0
$$

e il piano improprio, il quale è il piano tangente alla superficie lungo tutta la generatrice cuspidale, che in questo caso è la retta impropria del piano $x=0$. Saranno quindi invarianti rispetto al gruppo $G_{\text {xxxiv }}$ i complessi costituiti ciascuno dalle rette che intersecano la rigata cubica e il piano improprio secondo quaterne di punti proiettivi ad una data. Questi complessi sono del $6^{\circ}$ grado e, nel caso del gruppo da noi scelto a rappresentante del tipo, sono dati da

[XXXIV] $\left[2 z_{1} z_{2}^{2}-z_{2} z_{4}\left(3 z_{5}+1\right)+z_{4}^{3}\right]^{2}=b\left[3 z_{4}^{2}-2 z_{2}\left(3 z_{5}+1\right)\right]^{3}$.

Il complesso cubico e il complesso quadratico che, contati due o tre volte rispettivamente, si ottengono in codesto fascio per $h=0, \infty$, sono dati dalle rette che segano la rigata cubica e il piano fisso secondo quaderne di punti rispettivamente armoniche ed equianarmoniche; e il complesso quadratico appartiene alla caratteristica [(32I)]. Se poi si pone $b=-1 / 2$, il complesso XXXIV si spezza nel complesso lineare speciale (contato due volte) delle rette incidenti alla generatrice doppia e nel complesso del $4^{\circ}$ grado delle tangenti alla rigata cubica. 
VII.

Tabella dei complessi che ammettono un gruppo proiettivo integrabile.

24. Raccolgo in una tabella $i$ rentisei tipi di complessi che ammettono un gruppo proiettivo integrabile, ordinandoli secondo il numero dei parametri del gruppo corrispondente. A facilitare eventuali rafftonti, mettero accanto a ciascun tipo lo stesso numero ordinale che esso ebbe nelle pagine precedenti e contrassegnerò codesto numero con un asterisco, ogni qualvolta il tipo sarà duale di sè stesso.

Gruppo a cinque parametri:

$[\mathrm{X}]$

$$
\left\{p, q, \quad z_{4}=b z_{2}^{c} U, x p+c y q \quad(c \neq 0, \pm 1,2,1 / 2)\right.
$$

$[\mathrm{XI}]$

$$
\left\{\begin{array}{c}
z_{1}=b e^{\gamma_{2}} \\
p, \quad q, \quad r, \quad U, \quad z p+y q
\end{array}\right.
$$

$[\mathrm{XIII}]^{*}$

$$
\left\{\begin{array}{c}
2 z_{+}\left(z_{s}+1\right)+z_{2}^{2}=0 \\
r, p-y r, q+x r, q+y p, \quad 2 x p+y q+3 z r .
\end{array}\right.
$$

Gruppo a quattro parametri:

[IX]

$[\mathrm{XIV}]^{*}$

$$
\left\{\begin{array}{l}
\varphi\left(z_{2}, z_{4}\right)=0 \\
p, \quad q, \quad r, \quad U
\end{array}\right.
$$$$
\left\{\begin{array}{c}
z_{2}^{c}=b z_{4}\left(z_{5}+\mathrm{I}\right) \\
r, \quad p-y r, \quad q+x r, \quad c x p+y q+(c+\mathrm{I}) z r
\end{array}\right.
$$

$[\mathrm{XV}]^{*}$

$$
\left\{\begin{array}{c}
z_{s}+\mathrm{I}=b z_{2} e^{-\frac{z_{4}}{z_{2}}} \\
r, p-y r, q+x r, z r-x q+U
\end{array}\right.
$$

[XVI]

$$
\left\{\begin{array}{c}
z_{4}=b z_{2} e^{\frac{z_{5}+1}{z_{2}}} \\
r, p-y r, q+x r, \quad x p+z r-q
\end{array}\right.
$$

[XVIII]*

$$
\left\{\begin{array}{c}
z_{2}=b z_{s}^{c} \\
r, \quad x q, \quad x p+z r, \quad(c-\mathrm{I}) y q+c z r
\end{array}\right.
$$

[XIX]

$$
\left\{\begin{array}{c}
z_{2}=b e^{\frac{z_{5}}{z_{2}}} \\
r, \quad x q, \quad x p+z r, \quad q-x p
\end{array}\right.
$$

$$
\left\{\begin{array}{c}
z_{2} z_{5}-z_{4}=0 \\
r, \quad p+x r, \quad y q, \quad x p+2 z r .
\end{array}\right.
$$


Gruppo a tre parametri:

$[\mathrm{XII}]^{*}$

$$
\left\{\begin{array}{c}
\varphi\left(\frac{z_{2}}{z_{5}+1}, \frac{z_{4}}{z_{5}+1}\right)=0 \\
r, p-y r, q+x r
\end{array}\right.
$$

$[\mathrm{XVII}]^{*}$

$$
\left\{\begin{array}{c}
\varphi\left(z_{2}, z_{s}\right)=0 \\
r, x q, x p+z r
\end{array}\right.
$$

$[\mathrm{XXI}]^{*}$

$$
\left\{\begin{array}{c}
\left(z_{2} z_{5}-z_{4}\right) z_{4}^{c+1}=b z_{2}^{c+3} \\
r, \quad p+x r, \quad y q+c(z r+U)
\end{array}\right.
$$

[XXII]

$$
\left\{\begin{array}{c}
z_{4}=b z_{2} e^{\frac{z_{2} z_{5}-z_{4}}{z_{2}^{2}}} \\
r, p+x r, q+x p+2 z r
\end{array}\right.
$$

[XXIII] $]^{*}$

$$
\left\{\begin{array}{c}
z_{4}=b z_{2} e^{\frac{z_{2} z_{5}-z_{4}}{z_{2} z_{4}}} \\
r, \quad p+x r, p+y q
\end{array}\right.
$$

[XXIV]

$$
\left\{\begin{array}{c}
z_{2}^{a-1} z_{5}=b\left(z_{4}^{2}-2 z_{2}\right)^{\frac{a}{2}} \\
r, \quad x q+y r, z r-x p+a U
\end{array}\right.
$$

[XXV]

$$
\left\{\begin{array}{c}
2 z_{5}=b z_{2} e^{\frac{z_{4}^{2}-2 z_{2}}{z_{2}^{2}}} \\
r, x q+y r, \quad x p-z r+q
\end{array}\right.
$$

$[\mathrm{XXVI}]^{*}$

$$
\left\{\begin{array}{c}
z_{2}^{a-1} z_{5}=b e^{\frac{z_{4}}{z_{2}}} \\
r, \quad z r+a U, \quad x q+U
\end{array}\right.
$$

$[\mathrm{XXVII}]^{*}$

$$
\left\{\begin{array}{c}
z_{2}^{a+b} z_{5}=b z_{4}^{b+r} \\
r, z r+a U, \quad y q+b U
\end{array}\right.
$$

[XXVIII]

$$
\left\{\begin{array}{c}
z_{4}^{a+1}=b z_{2}^{a} e^{\frac{z_{5}}{z_{2}}} \\
r, q+z r, \quad x p+a p
\end{array}\right.
$$

$[\mathrm{XXIX}]$

$$
\left\{\begin{array}{c}
z_{2}^{2}=b e^{\frac{2 z_{2} z_{5}-a z_{4}^{2}}{z_{2}^{2}}} \\
r, q+z r, \quad x q+a p
\end{array}\right.
$$

$[\mathrm{XXX}]^{*}$

$$
\left\{\begin{array}{c}
z_{2}^{2(c-\mathrm{I})}=b z_{4}^{2}\left[4 z_{2} z_{3}+\left(z_{s}+\mathrm{I}\right)^{2}\right]^{c-2} \\
p-y r, q-x r, \quad(c-1) x p+y q+c z r
\end{array}\right.
$$

$[\mathrm{XXXI}]^{*}$

$$
\left\{\begin{array}{c}
z_{2}^{2}=b z_{4}^{2} e^{\frac{4 z_{2} z_{3}+\left(z_{5}+1\right)^{2}}{z_{2} z_{4}}} \\
p-y r, q-x r, r+y q-x p
\end{array}\right.
$$


$[\mathrm{XXXII}]^{*}$

$$
\left\{\begin{array}{l}
z_{1} z_{4}=b z_{2} z_{3} \\
x p, \quad y q, \quad z^{r}
\end{array}\right.
$$

[XXXIII]*

$$
\left\{\begin{array}{c}
\left(1-2 z_{1} z_{2}\right) z_{4}^{2}=b\left(z_{2} z_{3}-z_{4}\right)^{2} \\
p+x r, y q, \quad x p+2 z^{r}
\end{array}\right.
$$

$[\mathrm{XXXIV}]^{*}\left\{\begin{array}{c}{\left[2 z_{1} z_{2}^{2}-z_{2} z_{4}\left(3 z_{5}+\mathrm{I}\right)+z_{4}^{3}\right]^{2}=b\left[3 z_{4}^{2}-2 z_{2}\left(3 z_{5}+1\right)\right]^{3}} \\ p+2 x q+3 y r, q+3 x r, x p+2 y q+3 z r .\end{array}\right.$

Modena, dicembre 1906.

Ugo AmALD. 